(6)

\section{OPEN ACCESS}

${ }^{1}$ Institute of Cardiovascular

Sciences, University of

Birmingham, Birmingham, UK

${ }^{2}$ SWBH NHS Trust,

Birmingham, UK

${ }^{3}$ UHB NHS Trust, Birmingham, UK

${ }^{4}$ Atrial Fibrillation NETwork

(AFNET), Münster, Germany

${ }^{5}$ Department of Cardiovascular

Medicine, Hospital of the

University of Münster, Münster,

Germany

\section{Correspondence to}

Professor Paulus Kirchhof, Institute of Cardiovascular

Sciences, Institute for

Biomedical Research, 1st floor,

Room no. 136, Vincent Drive,

Birmingham B15 2TT, UK:

p.kirchhof@bham.ac.uk,

PKirchhofPA@contacts.bham.

ac.uk

Received 1 October 2015 Revised 26 November 2015 Accepted 26 November 2015

Published Online First

20 January 2016

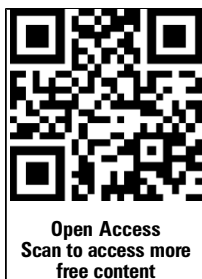

CrossMark

To cite: Jawad-UI-

Qamar M, Kirchhof P. Heart

2016;102:573-580.

\title{
Almanac 2015: atrial fibrillation research in Heart
}

\author{
Muhammad Jawad-UI-Qamar, ${ }^{1,2}$ Paulus Kirchhof 1,2,3,4,5
}

\begin{abstract}
Atrial fibrillation continues to attract interest in the cardiovascular community and in Heart. Over 60 original research and review papers published in Heart in 20142015 cover various aspects of atrial fibrillation, from associated conditions and precipitating factors to new approaches to management. Here, we provide an overview of articles on atrial fibrillation published in Heart in 2014-2015, highlighting new developments, emerging concepts and novel approaches to treatment.
\end{abstract}

\section{INTRODUCTION}

The 2014-2015 have been active years in atrial fibrillation (AF) research. Contributions from Heart on this subject have been very significant and substantial. The articles reflect the diverse nature of problems that patients with $\mathrm{AF}$ and their physicians are faced with, ranging from factors that allow to estimate the risk of cardiovascular complications and novel, hypothesis-generating associations of $\mathrm{AF}$ with biomarkers to insights into the optimal approach to anticoagulation, rate control and rhythm control therapy. We summarise some of the more interesting findings reported on AF in Heart in the year 2014 and 2015.

\section{RISK FACTORS FOR DEVELOPING AF AND AF PROGRESSION}

Baseline ECG, heart rate and age

A US-based registry (ORBIT -AF Outcomes Registry for Better Informed Treatment of Atrial Fibrillation.) analysed more than 6000 patients for risk of progression of AF from paroxysmal to persistent to permanent. ${ }^{1}$ It was found that increasing age by 10 (OR 1.16) and presence of AF in ECG at baseline (OR 2.30) were strong predictors of AF progression. Meanwhile, decreasing heart rate than 80 (OR 0.84) was protective against progression of $\mathrm{AF}$.

\section{Ivabradine}

An important meta-analysis identified a small (relative risk (RR) 1.15) but relevant risk for developing AF in patients treated with ivabradine. ${ }^{2}$ This effect is incidentally observed in the same group of patients (baseline heart rate $>70$ ) that get the highest benefit from receiving ivabradine in terms of decreasing hospitalisation. This RR of developing AF could be attributed to change in the $I_{f}$ current induced by ivabradine, a modification of the atrial resting membrane potential, or due to the potential proarrhythmic effects of bradycardia. Mechanistic studies are warranted to identify the mechanisms of $\mathrm{AF}$ induction by ivabradine (figure 1).

\section{Diastolic dysfunction}

Results of subanalysis from Tromsø Study in Norway showed that severely enlarged atrial size as a marker for diastolic dysfunction was only echocardiographic marker associated with risk of developing AF (HR 4.2). ${ }^{3}$ This was independent of other mitral valve Doppler indices of diastolic dysfunction. Left atrial (LA) size increases with increasing diastolic dysfunction due to long-term change in left heart flow dynamics.

\section{Haemodialysis}

A hypothesis-generating analysis of a cohort of dialysis patients with an implanted pacemaker or defibrillator suggested that the haemodialysis procedure itself could trigger $\mathrm{AF}^{4}$ The onset of atrial high-rate events (AHRE) clusters around the time of dialysis that can be considered as a proxy measure for AF. An association between higher exacted volume and lower dialysate potassium concentration is considered as triggers of AF. Comparatively, patients receiving peritoneal dialysis had less episodes of AF. These findings are consistent across other studies, which includes age, gender, coronary artery disease and atrial dimensions as additional risk for developing $\mathrm{AF}$ in haemodialysis patients (figure 2). ${ }^{56}$

\section{Exercise}

Several papers have assessed the impact of physical exercise on risk of developing $\mathrm{AF}^{7}{ }^{8}$ There was an interesting Swedish study in more than 44000 healthy adult men demonstrating a U-shaped relation between exercise and risk of $\mathrm{AF}^{9}$ They found that moderate-to-severe exercise in leisure time or bicycling/walking for more than $60 \mathrm{~min}$ in a day was associated with a RR of 1.17 and 1.04 of developing AF later in life. Interestingly, this trend is seen to reverse in the older age with reduction in RR with similar levels of exercise. Similar favourable trends of exercise are seen for middle-aged and elderly women. ${ }^{10}$

It is well established that exercise capacity is reduced in $\mathrm{AF}$ with or without the presence of left ventricular systolic impairment. ${ }^{11}{ }^{12}$ There is a suggestion that paroxysmal atrial fibrillation (PAF) could add to the interplay between exercise and inherited conditions such as hypertrophic cardiomyopathy. ${ }^{13}$ Patients with PAF had a substantially lower exercise tolerance, even though they remained in Sinus Rhythm (SR) during exercise testing. After adjustment for age, sex and body mass index, PAF still had an independent RR of 4.65 for reduced exercise tolerance.

\section{No benefit of atrial septal defect closure}

A Danish study for adult patients diagnosed with Atrial septal defect (ASD) shows that ASD closure gives rise to a higher risk of new AF (HR 8.4) as compared with age and gender matched comparison cohort, with a 10 year cumulative incidence of $11 \% .^{14}$ The risk of stroke was higher in patients 


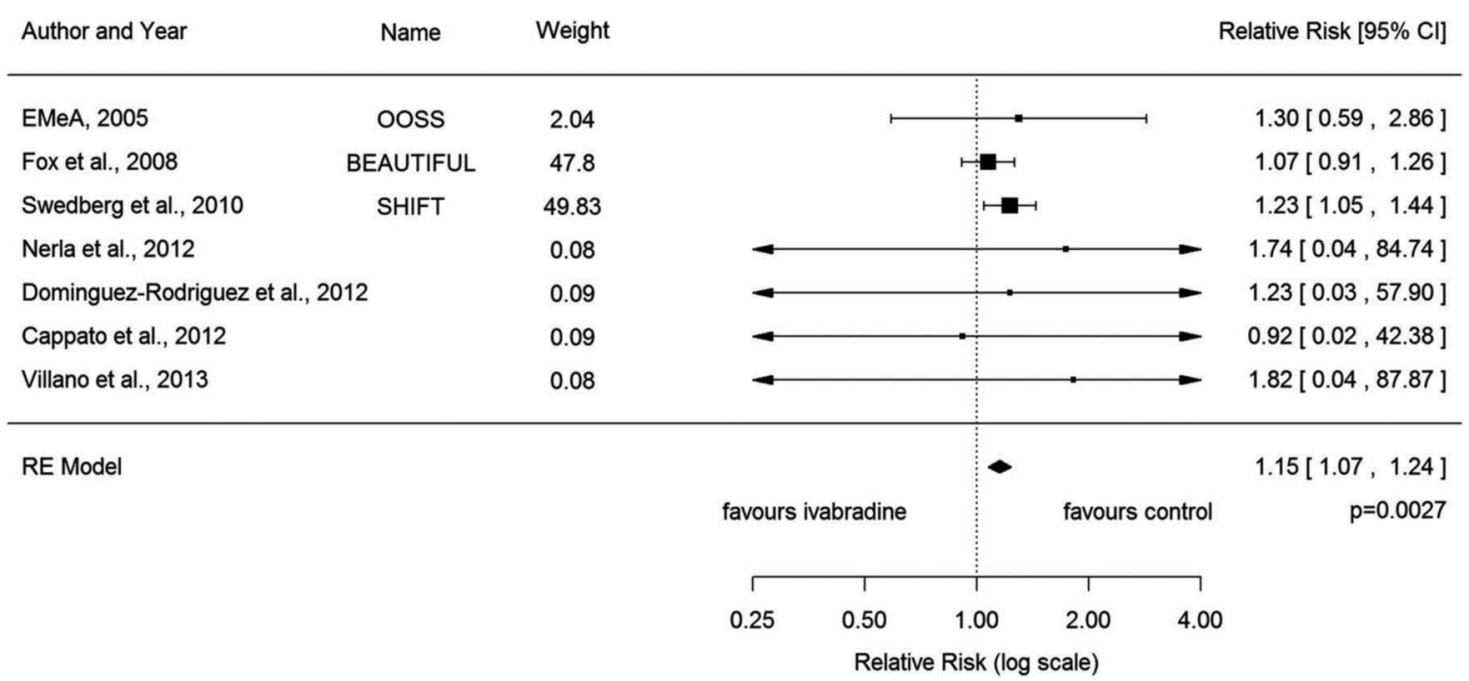

Figure 1 Forest Plot of RR of atrial fibrillation with ivabradine. ${ }^{2} \mathrm{RR}$, relative risk.

with ASD with (HR 2) or without (HR 2.6) ASD closure, suggesting that $\mathrm{AF}$ is not prevented by ASD closure. This observation suggests that other factors than the altered haemodynamic function determine $\mathrm{AF}$ in patients with ASD. Alternatively, an even earlier timing of ASD closure may be needed to prevent AF.

\section{Other hypothesis-generating associations with AF}

An analysis of Myocardial Ischemia National Audit Project database covering all PCI Percutaneous Coronary Intervention procedures in England and Wales for the short-term effects of air pollution on cardiovascular events in England and Wales was published in 2014. ${ }^{15}$ This showed an increased risk of hospital admissions due to $\mathrm{AF}$ and arrhythmias and high levels of $\mathrm{NO}_{2}$. A high content of particulate matter less than $2.5 \mu \mathrm{m}$ in diameter was associated with increased mortality secondary to AF, arrhythmia and pulmonary embolism.

An observational study for risk of developing atrial flutter and fibrillation in patients admitted with pericarditis showed an incidence rate of $4.3 \%$ with more than $90 \%$ having an episode of $\mathrm{AF}$ in the first $24 \mathrm{~h} .{ }^{16}$ All of them reverted to sinus rhythm. However, there was $35 \% 3$ month recurrence rate for patients who initially developed AF. The authors advocated for anticoagulation in high-risk patients. No increased risk of pericardial tamponade with anticoagulant therapy was shown.

\section{BIOMARKERS IN AF}

Biomarkers have recently generated a lot of interest in prediction, diagnosis and prognostic risk stratification of $\mathrm{AF}^{17-20}$

\section{Troponin I, natriuretic peptides and norepinephrine levels}

An substudy of RE-LY (Randomized Evaluation of Longterm anticoagulant TherapY) regarding the prognostic value and risk stratification of biomarkers in $\mathrm{AF}$ has indicated an interesting use of cardiac biomarkers. ${ }^{21}$ They have indicated that serial high levels of cardiac troponin I (cTnI) and N Terminal-pro Brain Natriuretic Peptide (NT-proBNP) are associated with high incidence of stroke and systemic embolism (HR 4.54) and vascular death (HR 8.62). Others have earlier found similar association between these biomarkers and prognosis in $\mathrm{AF}^{22-25}$ A Japanese study showed higher levels of atrial natriuretic peptide, brain natriuretic peptide and norepinephrine (NE) in persistent versus PAF. $^{26}$ Only elevated NE levels were found to have an association with sick sinus syndrome (figure 3).

\section{Liver enzymes}

Elevated circulating levels of liver enzymes are found to have moderately strong association with increased $\mathrm{AF}$ incidence in a large prospective community-based cohort study of more than 15000 subjects. ${ }^{27}$ The association was linear and strongest for gamma glutamyl transferase (GGT) with doubling of GGT levels leading to $20 \%$ increase in $\mathrm{AF}$ risk after adjusting for the confounding factors. The association of $\mathrm{AF}$ incidence and aspartate amino transferase (AST) and to a lesser extent alanine amino transferase (ALT) showed a U-shaped curve with maximum incidence at the two extremes. This can be explained by right-sided heart failure leading to hepatic congestion or non-alcoholic fatty liver disease that increases the cardiovascular risk due to effect on glucose and lipid metabolism ${ }^{28-30}$ Correlation between deranged LFTs and risk of cardiovascular disease has also been published in a subanalysis from Framingham heart study. ${ }^{31}$

\section{Adiponectin}

An association as a function of increasing age was seen between higher circulating adiponectin levels and risk of developing

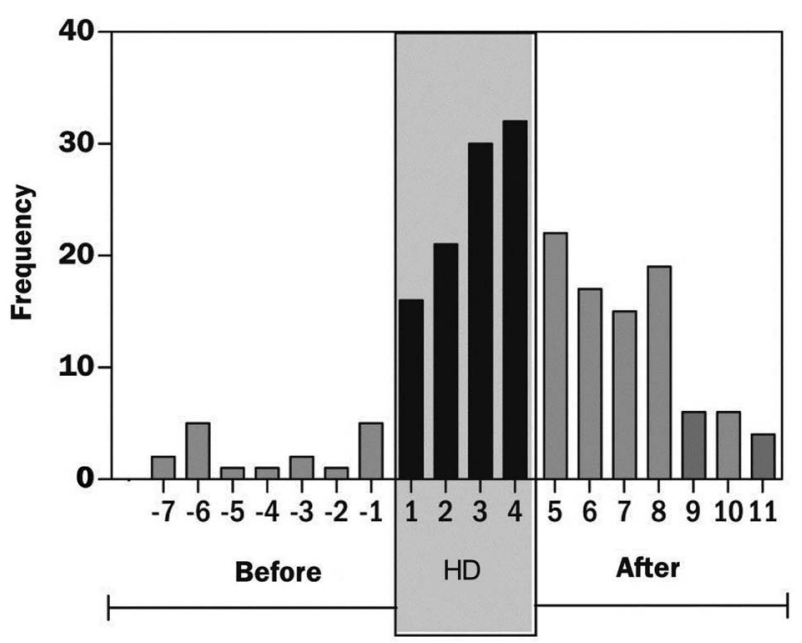

Hours after the start of the dialysis procedure

Haemodialysis

\section{Before/After}

haemodialysis

Figure 2 Onset of atrial fibrillation episodes on relation to start of haemodialysis. $^{4}$ 

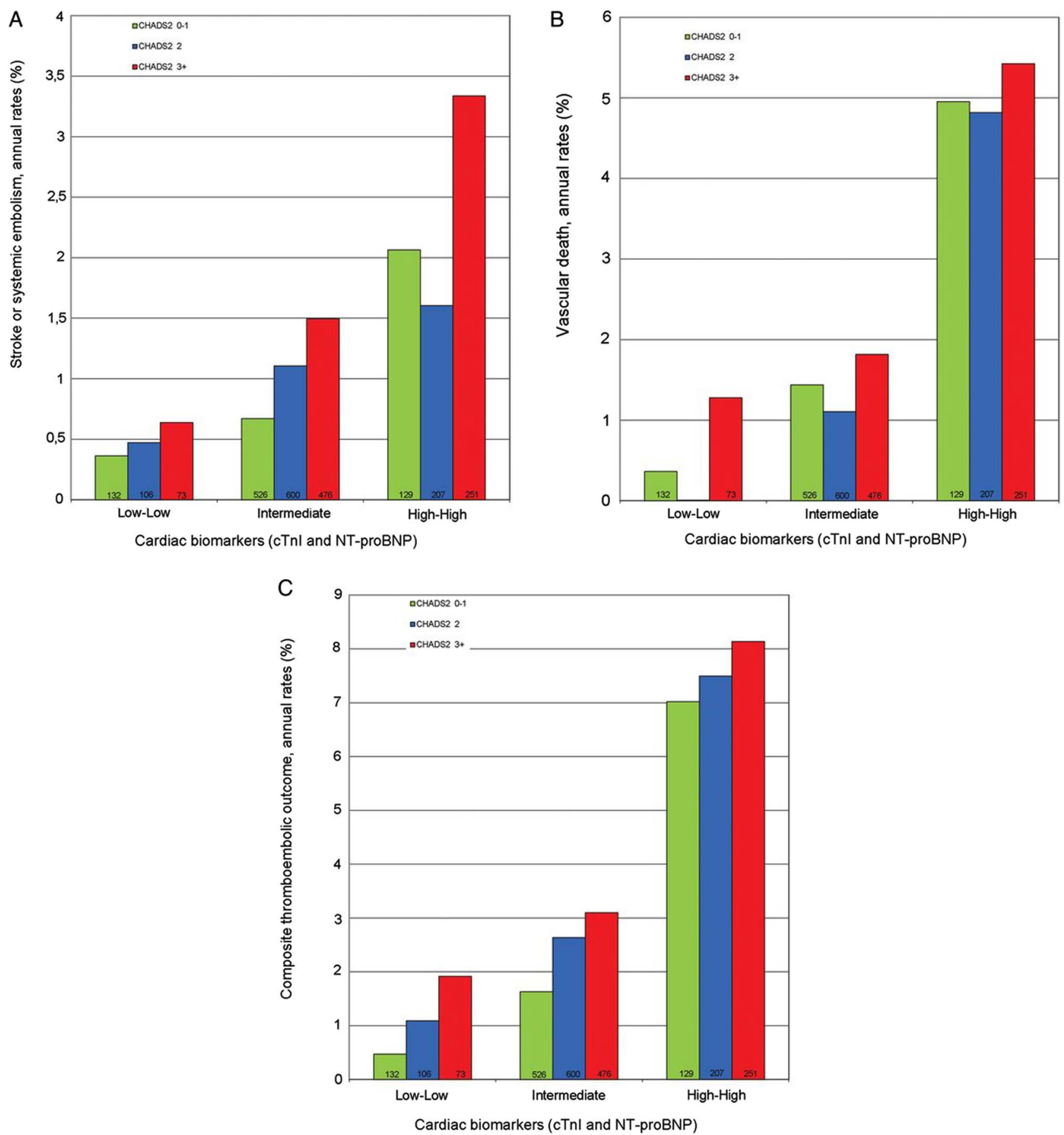

Figure 3 Study endpoints in relation to combined cardiac biomarker levels and $\mathrm{CHADS}_{2}$ score. Stroke or systemic embolism (A), vascular death (B) and composite thromboembolic outcome consisting of ischaemic stroke, systemic embolism, myocardial infarction, pulmonary embolism and vascular death (C). ${ }^{21}$ cTnl, cardiac troponin. NT-pro BNP, N Terminal-pro Brain Natriuretic Peptide. CHADS2, Stroke score (based on a 1 point each for Congestive heart failure, Hypertension, Age more than 75, Diabetes mellitus and 2 points for previous Stroke or TIA.)

$\mathrm{AF}^{32}$ This is paradoxical to the contemporary belief that higher adiponectin levels are cardioprotective. Further work is required in this field to identify a clear association with this novel biomarker.

\section{EPIDEMIOLOGY AND RISK FACTORS} Increasing incidence of AF in the UK

A large population-based cohort study looked at the discharge record of 2.2 million individuals aged 45 and above, from UK Clinical Practice Research Datalink. ${ }^{33}$ They found more than 91000 incident AF cases. The incidence of AF has increased from $5.9 / 1000$ person-years in 2001 to $6.9 / 1000$ person-years in 2013. The overall incidence in terms of 1000 person-years increases with increasing age (25.1 for patients between 80-89) and is also higher for Caucasians (8.1) versus Asians (5.4) and African Americans (4.6).

Predictive value of the $\mathrm{CHADS}_{2}$ and $\mathrm{CHA}_{2} \mathrm{DS}_{2}$ Vasc scores for cardiovascular events in patients without $\mathrm{AF}$

$\mathrm{CHADS}_{2}$ and $\mathrm{CHA}_{2} \mathrm{DS}_{2}$ Vasc scores have been validated to predict the risk of stroke in patients with $\mathrm{AF}^{34}{ }^{35}$ In a study, these scores were used to assess risk of new stroke/transient 
A

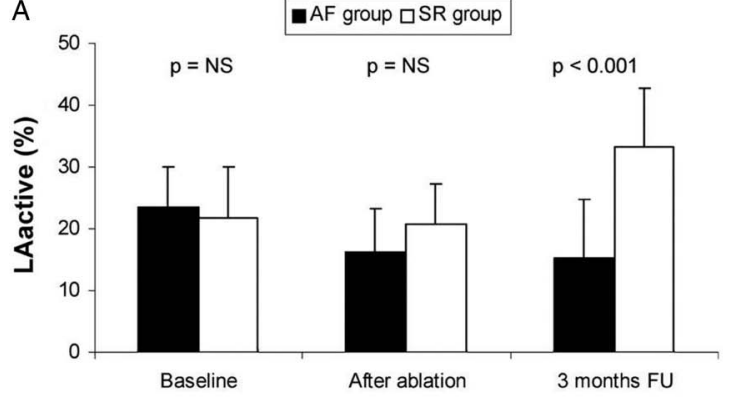

B

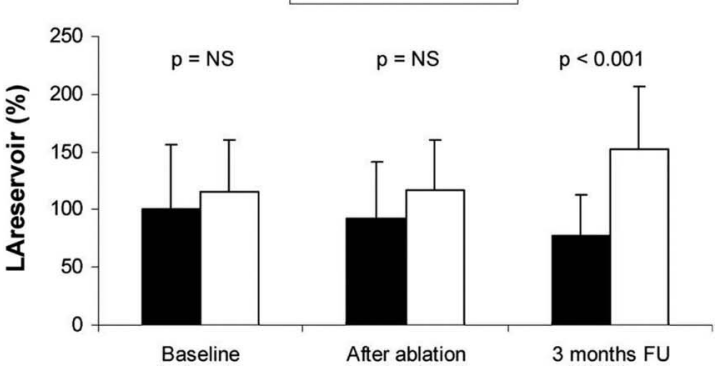

Figure 4 Changes in LA function after catheter ablation. SR group shows an improvement in LA reservoir function. AF, atrial fibrillation; LA, left atrial; NS, not significant.

ischaemic attack (TIA) in the absence of AF in patients with an acute coronary syndrome (ACS). ${ }^{36}$ Both these scores showed a reasonable association predicted annual risk of stroke/TIA with an absolute annual incidence of $1 \%$ with $\mathrm{CHADS}_{2} \geq 3$ and $\mathrm{CHA}_{2} \mathrm{DS}_{2}$ Vasc $\geq 4$. This is in line with the results from another study by Poci et $a l^{37}$ that found association of $\mathrm{CHADS}_{\mathrm{s}}$ with mortality and stroke. Others have shown association of these score with mortality after stroke, risk of developing new AF and risk of stroke or death after CABG. ${ }^{38-40}$

\section{$\mathrm{AF}$ and heart failure}

$\mathrm{AF}$ and heart failure are often 'vicious twins', and each condition can worsen the other. A study in Tanzania showed that AF attributed to at least $16 \%$ cases of clinical heart failure presenting to tertiary-care hospital. ${ }^{41}$ This is an interesting finding if compared with the EPOCH (Epidemiology, Practice, Outcomes, and Costs of Heart Failure) study conducted in the USA in 2004 that compared epidemiological characteristics of hospitalised patients with heart failure and association of various comorbidities with different ethnicities, which showed AF was prevalent in $19.7 \%$ of African-Americans with heart failure as compared with $38.3 \%$ in Caucasian patients with heart failure. ${ }^{42}$ The report illustrates the global impact of $\mathrm{AF}$ and heart failure.

\section{AF as a predictor of increased mortality in low-gradient aortic stenosis}

AF was found as independent predictor of mortality (HR 1.74) in patients with aortic stenosis (AS) in a large single centre observational study of severe AS treated with medical therapy, surgical aortic valve replacement or transcatheter aortic valve implantation. $^{43}$ In general, the group receiving medical treatment had the worst prognosis with all-cause mortality of $81 \%$ at 3.9 years of follow-up. A study showed AF as a determinant of low-flow state in severe AS (OR 4.17). ${ }^{44}$ In another study, patients with severe low-gradient AS, AF was also associated with a poor prognosis and increased mortality. ${ }^{45}$ These observations highlight the importance to diagnose AF in patients with AS.

\section{Overall AF prevalence in hospitalised patients}

An interesting cross-sectional survey in Belgium on a single day in a tertiary-care hospital identified total prevalence of $\mathrm{AF}$ at $16.8 \%{ }^{46}$ The presence of AF was associated with old age, hypertension and valvular heart disease. Interestingly, only $51 \%$ were appropriately treated with oral anticoagulation.

\section{Prognosis of silent AF after myocardial infarction}

An observational study by Stamboul et al shows worse 1 year prognosis with increased hospital admissions and worsening heart failure for patients found to have silent AF within 2 days of admission with acute myocardial infarction (MI). ${ }^{47}$ It was previously suggested that silent $\mathrm{AF}$ was three times more common than symptomatic AF after acute MI. ${ }^{48}$ This highlights importance of continuous ECG monitoring for all patients with MI to detect AF.

\section{SCREENING FOR SILENT AF}

Early diagnosis of AF is highly desirable to initiate therapy prior to the first complication. ${ }^{49} \mathrm{~A}$ review article sheds light on emerging techniques to screen for $\mathrm{AF}^{50}$ Patient-operated ECG monitors or smartphone sensors may be suitable tools to screen for $\mathrm{AF}^{51-54}$ In selected patients, long-term screening with

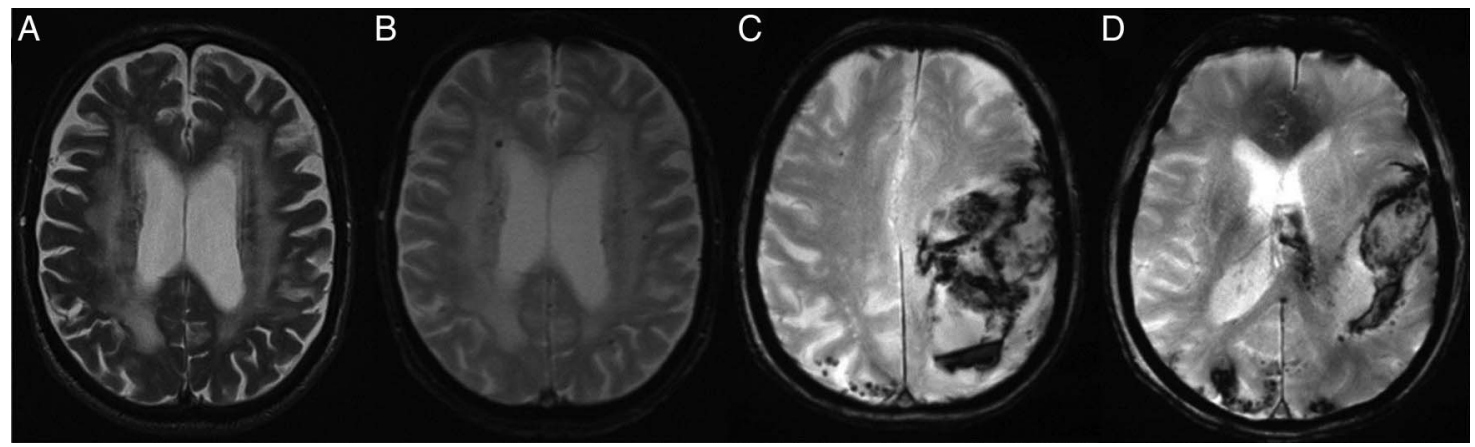

Figure 5 Patients with atrial fibrillation deemed unsuitable for oral anticoagulation T2 (A) and T2* (B) image at $1.5 \mathrm{~T}$ demonstrating confluent white matter hyperintensities and cerebral microbleeds in a patient with atrial fibrillation with recent transient ischaemic attack T2* images (C/D) at 3.0 T demonstrating an acute lobar haemorrhage and multiple cerebral microbleeds in a patient with atrial fibrillation with suspected cerebral amyloid angiopathy. ${ }^{59}$ 


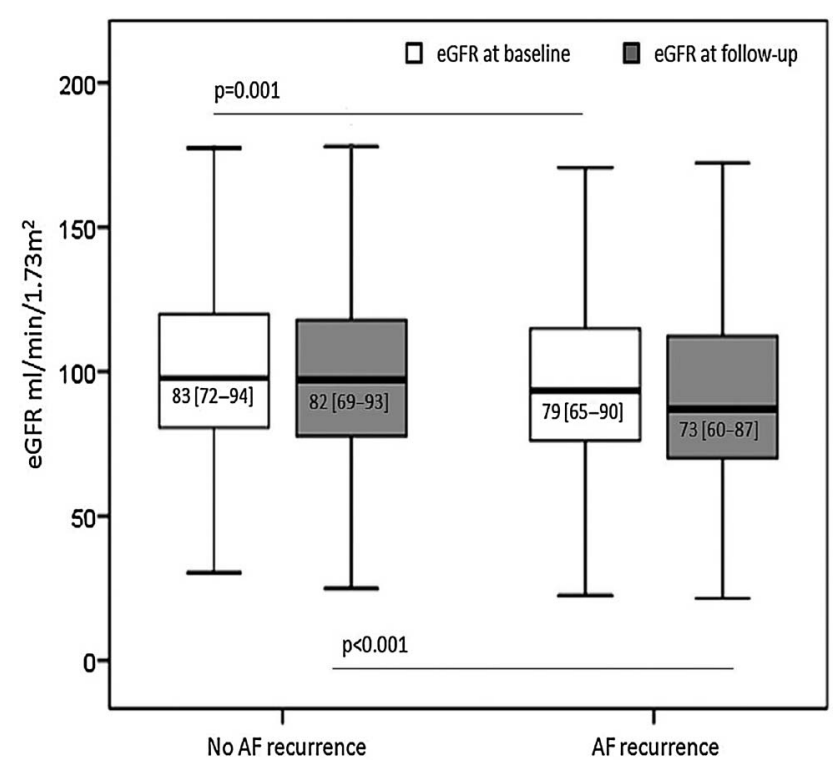

Figure 6 Estimated GFR in patients with and without recurrences at baseline and follow-up. ${ }^{76} \mathrm{AF}$, atrial fibrillation.

implantable loop recorder can generate more AF detection. ${ }^{55}$ The need for AF-screening programmes, their optimal design and the best use of therapy (mainly oral anticoagulation) are not fully understood. Ongoing studies evaluating initiation of anticoagulation in patients with atrial high-rate episodes (eg, ARTESiA or NOAH-AFNET 6) and community screening programmes such as STROKESTOP ${ }^{5657}$ will provide further information in future.

\section{IMAGING IN AF}

\section{Prognostic factors based on imaging parameters}

A review article highlights several adverse prognostic features in imaging for $\mathrm{AF}^{58}$ In two-dimensional conventional echocardiography, LA dilatation and left ventricular systolic function was shown to be associated with increased risk of stroke, heart failure and all-cause mortality. Mitral stenosis and hypertrophic cardiomyopathy (HCM) strongly also increases the risk of stroke in AF. In trans-oesophageal echocardiography (TOE), spontaneous echo contrast, LA thrombus and complex aortic valve $(\mathrm{AV})$ plaque predict increased risk of systemic thromboembolism and stroke. In cardiac MRI (CMRI), favourable parameters were associated with decreased risk of stroke (OR 0.2). Moreover, LA conduit function as measured by CMRI was an important measure of success after catheter ablation (figure 4).

\section{Brain MRI to tailor anticoagulation therapy}

Another review article looked into the value of brain MRI imaging to tailor anticoagulation therapy. ${ }^{59}$ It was found that silent brain infarction is commonly seen in patients with $\mathrm{AF}^{60}{ }^{61}$ This is found to be dependent on the age of patient and on the type of AF. Presence of cerebral microbleeds (CMB), which are frequently found in patients with $\mathrm{AF}$ without prior stroke, should be taken as a risk for intracranial bleeding on anticoagulation. However, large studies are needed to confirm this observation. Number and location of CMB is affected by $\mathrm{CHADS}_{2}$ and $\mathrm{CHA}_{2} \mathrm{DS}_{2}$ Vasc scores. This is mostly due to effect of vascular risk factors (figure 5). ${ }^{62}$

\section{Body Surface Potential Mapping and ECG imaging in AF}

Use of ECG imaging, a novel fibrillation imaging modality using surface ECG combined with cardiac CT/MRI for the atrial anatomy to assess complexity of AF, was also highlighted. ${ }^{63}$ More recently, Body Surface Potential Mapping has been shown to identify the high-frequency sources in the atria without an imaging modality that were previously measured by invasive methods. ${ }^{64}$ This can lead to better patient selection before the actual ablation procedure based on the prediction of response to AF ablation therapy.

\section{ANTICOAGULATION FOR STROKE PREVENTION Which antithrombotic therapy: NOACs, vitamin K antagonist, combination therapy}

A large network meta-analysis of 20 studies combining more than 78000 patients demonstrated that the new oral anticoagulants performed better in reducing the risk of stroke or venous embolism. ${ }^{65}$ Another meta-analysis with more than 100000
Figure 7 Temporal trends of imaging usage before (CT, MRI, TOE) and during (ICE) ablation. ${ }^{79}$ ICE, intracardiac echocardiography.

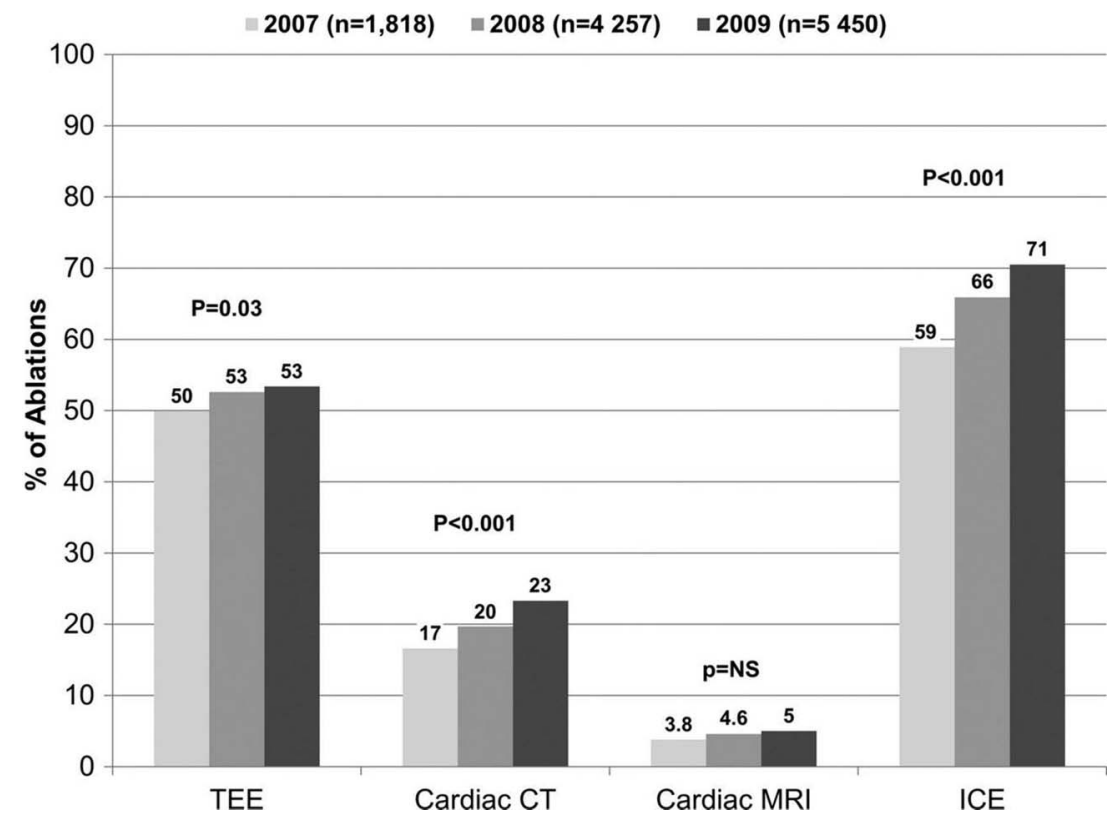




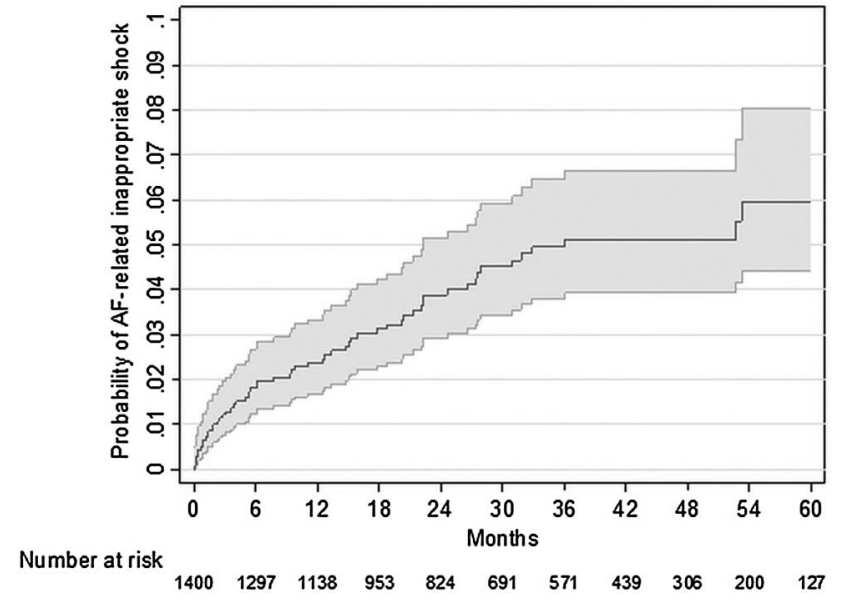

Figure 8 Kaplan-Meier estimation of probability of atrial fibrillation-related (AF-related) inappropriate shocks. ${ }^{89}$

patients showed $47 \%$ and $64 \%$ odds risk reduction of fatal bleeding with NOAC compared with vitamin $\mathrm{K}$ antagonist (VKA) therapy and low-molecular weight heparin (LMWH). ${ }^{66}$

It is well established that combined antiplatelet therapy and anticoagulation increases the risk of bleeding and that such 'triple therapy' should be confined to short periods of time in selected patients with ACS and/or recent PCI. ${ }^{67}$ An analysis of large European data set identified a common mistake in antithrombotic therapy in patients with AF, that is, the continuation of aspirin therapy in patients with stable vascular disease. ${ }^{69}$ Far more than half of the patients subjected to combination therapy with aspirin and oral anticoagulation had no indication for combination therapy, putting them at unnecessary risk for bleeding.

The TIARA trial in 238 patients, comparing aspirin and anticoagulation in a cohort of patients with AF with moderate stroke risk and 'favourable' imaging characteristics on TOE, suggested that some patients may fare well without anticoagulation. ${ }^{70}$ They found that in the absence of LA thrombus on TOE, aspirin was non-inferior to VKA for all-cause mortality, stroke, ACS and major bleeding. Interestingly, TIA was not included as a primary end point, and seven patients suffered from TIA in the aspirin group. This highlights the challenges when considering individualised anticoagulation therapy in patients at low-to-moderate stroke risk. The results of this pilot study need validation by a larger scale trial.

\section{Anticoagulation in atrial flutter}

A review article published in 2015, taking into account 52 published articles, indicated that atrial flutter renders a higher risk of thromboembolic complications. ${ }^{71}$ Imaging in atrial flutter also reveals high prevalence of spontaneous echo contrast and thrombus in left atrial appendage (LAA). Within the limitations of the small published data sets, this analysis underpins the current practice to offer anticoagulation to flutter patients.

\section{ABLATION FOR AF \\ Surgical ablation}

A meta-analysis summarising the available information on surgical ablation for AF has been published. ${ }^{72}$ This extensive and first-of-its-kind meta-analysis suggests that surgical LA ablation is a safe and effective method of maintaining sinus rhythm for more than 1 year in patients with AF and undergoing concomitant cardiac surgery as compared with cardiac surgery alone.

\section{NOACs or warfarin in catheter ablation}

A meta-analysis of 14 observational data sets compared dabigatran and warfarin for risks of thromboembolic events and major bleeds. ${ }^{73}$ There was no significant difference found between the two groups for either of the two end points. However, a numerical difference of more thromboembolic events in dabigatran group versus warfarin $(0.55 \%$ dabigatran vs $0.17 \%$ warfarin, RR $1.78,95 \%$ CI 0.66 to $4.80, \mathrm{p}=0.26$ ) was seen.

Another meta-analysis of observational studies also showed a non-significant but higher trend of worse neurological outcomes with dabigatran versus warfarin. ${ }^{74}$ No significant difference between dabigatran and warfarin was found in another meta-analysis. ${ }^{75}$ There is a need for controlled trials of uninterrupted anticoagulation regimes in patients with $\mathrm{AF}$ ablation.

\section{Change in renal function associated with arrhythmia recurrence}

A German study with a cohort of 783 subjects found an associated with AF recurrence and worsening renal function (GFR). ${ }^{76}$ It was found that patients with recurrence had worse GFR on baseline and on follow-up. The higher $\mathrm{CHADS}_{2}$ and $\mathrm{CHA}_{2} \mathrm{DS}_{2}$ Vasc scores were associated with worse renal functions. Finally, those patients who had decline in their GFR had more AF recurrences. This effect was independent of the type of OAC used. AF is known to decrease renal function. ${ }^{77}$ This may be possibly due to embolic phenomenon or haemodynamic mechanisms related to AF. Ablation and restoration of sinus rhythm generally improves renal function. ${ }^{78}$ However, deterioration of renal function may contribute to recurrent AF. Further studies are clearly needed to validate this novel observation (figure 6).

\section{Peri-procedural imaging in catheter ablation}

An observational study found an association between periprocedural cardiac imaging such as TOE, intracardiac echocardiography (ICE), cardiac CT and MRI, and better outcomes in patients undergoing catheter ablation for $\mathrm{AF}^{79}$ They found that use of preprocedural cardiac CT/MR was associated with a lower risk of TIA/stroke at 6 months $(0.4 \%$ vs $0.9 \%$, adjusted HR 0.46). Use of ICE was found to be associated with higher risk of bleeding $(1.1 \%$ vs $0.7 \%$, adjusted HR 1.76), but also with a lower incidence of repeat ablation $(5.7 \%$ vs $8.5 \%$, adjusted HR 0.68). Interestingly, TOE was not found to affect procedural outcomes. The usefulness of cardiac CT and MRI in identifying LA thrombus in patients undergoing ablation has been found in other studies ${ }^{80} 81$ on top of their benefit in assessing cardiac anatomy and pulmonary vein size and location ${ }^{82-84}$ and chances of successful outcome (figure 7). ${ }^{85}$

\section{AF-RELATED INAPPROPRIATE SHOCKS IN PATIENTS WITH ICD}

It has been known that prolonged ECG monitoring with pacemakers and CRT-D have high detection rates of atrial high-rate episodes, ${ }^{86} 87$ especially those with home monitoring that decreases the time to detect AHRE. ${ }^{88}$ Most, but not all of these episodes reflect paroxysmal, often undiagnosed (silent) AF. In a study of 1404 patients, it was found that AF lasting for more than $10 \mathrm{~min}$ is detected in a quarter of the patients who received CRT-D. ${ }^{89}$ Roughly, three-quarters of all inappropriate arrhythmia detections were due to AF, with 60 patients (4\% of the total) receiving inappropriate shocks due to $\mathrm{AF}$ (2.69 patients/100 patient-years). Reprogramming of the shock criteria could have avoided many of these inappropriate 
shocks. Another French case report also explained the use of home monitoring to prevent in appropriate AF-related shocks (figure 8). ${ }^{90}$

In summary, $\mathrm{AF}$ remains one of the major topics of research published in Heart. We learned a lot, but there is a lot more to find out and study to improve outcomes in patients with $\mathrm{AF}$ in future.

Contributors Both authors prepared and reviewed the text and searched for relevant papers in Heart 2014 and 2015.

Funding The preparation of this article was partially supported by funds from Charité Berlin (MonDAFIS), BHF (FS/13/32/30324), and Leducq Foundation.

Competing interests None declared.

Provenance and peer review Commissioned; internally peer reviewed.

Open Access This is an Open Access article distributed in accordance with the terms of the Creative Commons Attribution (CC BY 4.0) license, which permits others to distribute, remix, adapt and build upon this work, for commercial use, provided the original work is properly cited. See: http://creativecommons.org/ licenses/by/4.0/

\section{REFERENCES}

1 Holmqvist F, Kim S, Steinberg BA, et al. Heart rate is associated with progression of atrial fibrillation, independent of rhythm. Heart 2015;101:894-9.

2 Martin RI, Pogoryelova 0, Koref MS, et al. Atrial fibrillation associated with ivabradine treatment: meta-analysis of randomised controlled trials. Heart 2014;100:1506-10.

3 Tiwari S, Schirmer $\mathrm{H}$, Jacobsen BK, et al. Association between diastolic dysfunction and future atrial fibrillation in the Tromso Study from 1994 to 2010. Heart 2015;101:1302-8.

4 Buiten MS, de Bie MK, Rotmans JI, et al. The dialysis procedure as a trigger for atrial fibrillation: new insights in the development of atrial fibrillation in dialysis patients. Heart 2014;100:685-90.

5 Zimmerman D, Sood MM, Rigatto C, et al. Systematic review and meta-analysis of incidence, prevalence and outcomes of atrial fibrillation in patients on dialysis. Nephrol Dial Transplant 2012:27:3816-22.

6 Atar I, Konas D, Acikel S, et al. Frequency of atrial fibrillation and factors related to its development in dialysis patients. Int I Cardiol 2006;106:47-51.

7 Elosua $\mathrm{R}$, Arquer A, Mont L, et al. Sport practice and the risk of lone atrial fibrillation: a case-control study. Int I Cardiol 2006;108:332-7.

8 Mont L, Tamborero D, Elosua R, et al. Physical activity, height, and left atrial size are independent risk factors for lone atrial fibrillation in middle-aged healthy individuals. Europace 2008:10:15-20.

9 Drca N, Wolk A, Jensen-Urstad M, et al. Atrial fibrillation is associated with different levels of physical activity levels at different ages in men. Heart 2014; 100:1037-42.

10 Drca N, Wolk A, Jensen-Urstad M, et al. Physical activity is associated with a reduced risk of atrial fibrillation in middle-aged and elderly women. Heart 2015;101:1627-30.

11 Lee $\mathrm{SH}$, Jung $\mathrm{JH}$, Choi SH, et al. Exercise intolerance in patients with atrial fibrillation: clinical and echocardiographic determinants of exercise capacity. J Am Soc Echocardiogr 2005; 18:1349-54.

12 Atwood JE, Myers JN, Tang XC, et al. Exercise capacity in atrial fibrillation: a substudy of the Sotalol-Amiodarone Atrial Fibrillation Efficacy Trial (SAFE-T). Am Heart J 2007;153:566-72.

13 Azarbal F, Singh M, Finocchiaro G, et al. Exercise capacity and paroxysmal atrial fibrillation in patients with hypertrophic cardiomyopathy. Heart 2014;100:624-30.

14 Nyboe C, Olsen MS, Nielsen-Kudsk JE, et al. Atrial fibrillation and stroke in adult patients with atrial septal defect and the long-term effect of closure. Heart 2015:101:706-11.

15 Milojevic A, Wilkinson P, Armstrong B, et al. Short-term effects of air pollution on a range of cardiovascular events in England and Wales: case-crossover analysis of the MINAP database, hospital admissions and mortality. Heart 2014;100:1093-8.

16 Imazio M, Lazaros G, Picardi E, et al. Incidence and prognostic significance of new onset atrial fibrillation/flutter in acute pericarditis. Heart 2015;101:1463-7.

17 Schnabel RB, Wild PS, Wilde S, et al. Multiple biomarkers and atrial fibrillation in the general population. PLOS ONE 2014;9:e112486.

18 Rosenberg MA, Maziarz M, Tan AY, et al. Circulating fibrosis biomarkers and risk of atrial fibrillation: The Cardiovascular Health Study (CHS). Am Heart J 2014;167:723-8.e2.

19 Hijazi Z, Oldgren J, Siegbahn A, et al. Biomarkers in atrial fibrillation: a clinical review. Eur Heart J 2013:34:1475-80.

20 Howlett PJ, Hatch FS, Alexeenko V, et al. Diagnosing paroxysmal atrial fibrillation: are biomarkers the solution to this elusive arrhythmia? Biomed Res Int 2015;2015:910267.
21 Hijazi Z, Oldgren J, Andersson U, et al. Importance of persistent elevation of cardiac biomarkers in atrial fibrillation: a RE-LY substudy. Heart 2014;100:1193-200.

22 Roldan V, Marin F, Diaz J, et al. High sensitivity cardiac troponin T and interleukin-6 predict adverse cardiovascular events and mortality in anticoagulated patients with atrial fibrillation. I Thromb Haemost 2012;10:1500-7.

23 Smith JG, Newton-Cheh C, Almgren P, et al. Assessment of conventional cardiovascular risk factors and multiple biomarkers for the prediction of incident heart failure and atrial fibrillation. J Am Coll Cardiol 2010:56:1712-19.

24 Psychari SN, Chatzopoulos D, Iliodromitis EK, et al. C-reactive protein, interleukin 6, and $\mathrm{N}$-terminal pro-brain natriuretic peptide following cardioversion of atrial fibrillation: is there a role of biomarkers in arrhythmia recurrence? Angiology 2011:62:310-16

25 Hijazi Z, Oldgren J, Andersson U, et al. Cardiac biomarkers are associated with an increased risk of stroke and death in patients with atrial fibrillation: a Randomized Evaluation of Long-term Anticoagulation Therapy (RE-LY) substudy. Circulation 2012;125:1605-16.

26 Yoshida K, Kaneshiro T, Ito Y, et al. Elevated plasma norepinephrine level and sick sinus syndrome in patients with lone atrial fibrillation. Heart 2015;101:1133-8.

27 Alonso A, Misialek JR, Amiin MA, et al. Circulating levels of liver enzymes and incidence of atrial fibrillation: the Atherosclerosis Risk in Communities cohort. Heart 2014:100:1511-16.

28 Naschitz JE, Slobodin G, Lewis RJ, et al. Heart diseases affecting the liver and liver diseases affecting the heart. Am Heart J 2000;140:111-20.

29 Targher G, Bertolini L, Poli F, et al. Nonalcoholic fatty liver disease and risk of future cardiovascular events among type 2 diabetic patients. Diabetes 2005:54:3541-6.

30 Mikolasevic I, Racki S, Zaputovic L, et al. Nonalcoholic fatty liver disease (NAFLD) a new risk factor for adverse cardiovascular events in dialysis patients. Med Hypotheses 2014;82:205-8.

31 Targher G, Day CP, Bonora E. Risk of cardiovascular disease in patients with nonalcoholic fatty liver disease. N Engl J Med 2010;363:1341-50.

32 Macheret F, Bartz TM, Djousse L, et al. Higher circulating adiponectin levels are associated with increased risk of atrial fibrillation in older adults. Heart 2015;101:1368-74.

33 Martinez C, Katholing A, Wallenhorst C, et al. Increasing incidence of non-valvular atrial fibrillation in the UK from 2001 to 2013. Heart 2015;101:1748-54.

34 Gage BF, Waterman AD, Shannon W, et al. Validation of clinical classification schemes for predicting stroke: results from the National Registry of Atrial Fibrillation. JAMA 2001;285:2864-70.

35 Lip GY, Nieuwlaat R, Pisters R, et al. Refining clinical risk stratification for predicting stroke and thromboembolism in atrial fibrillation using a novel risk factor-based approach: the euro heart survey on atrial fibrillation. Chest 2010;137:263-72.

36 Mitchell LB, Southern DA, Galbraith D, et al. Prediction of stroke or TIA in patients without atrial fibrillation using CHADS2 and CHA2DS2-VASC scores. Heart 2014;100:1524-30.

37 Poci D, Hartford M, Karlsson T, et al. Role of the CHADS2 score in acute coronary syndromes: risk of subsequent death or stroke in patients with and without atrial fibrillation. Chest 2012;141:1431-40.

38 Henriksson KM, Farahmand B, Johansson S, et al. Survival after stroke- the impact of CHADS2 score and atrial fibrillation. Int I Cardiol 2010;141:18-23.

39 Chao TF, Liu CJ, Chen SJ, et al. CHADS2 score and risk of new-onset atrial fibrillation: a nationwide cohort study in Taiwan. Int I Cardiol 2013;168:1360-3.

40 Hornero F, Martin E, Paredes F, et al. Stroke after coronary artery bypass grafting: preoperative predictive accuracies of CHADS2 and CHA2DS2VASC stroke risk stratification schemes. J Thorac Cardiovasc Surg 2012;144:1428-35.

41 Makubi A, Hage C, Lwakatare J, et al. Contemporary aetiology, clinical characteristics and prognosis of adults with heart failure observed in a tertiary hospital in Tanzania: the prospective Tanzania Heart Failure (TaHeF) study. Heart 2014;100:1235-41.

42 Ruo B, Capra AM, Jensvold NG, et al. Racial variation in the prevalence of atrial fibrillation among patients with heart failure: the Epidemiology, Practice, Outcomes, and Costs of Heart Failure (EPOCH) study. J Am Coll Cardiol 2004:43:429-35

43 Pilgrim T, Englberger L, Rothenbuhler $M$, et al. Long-term outcome of elderly patients with severe aortic stenosis as a function of treatment modality. Heart 2015:101:30-6.

44 Magne J, Mohty D, Boulogne C, et al. Prognosis importance of low flow in aortic stenosis with preserved LVEF. Heart 2015;101:781-7.

45 Moretti $M$, Fabris $E$, Morosin $M$, et al. Prognostic significance of atrial fibrillation and severity of symptoms of heart failure in patients with low gradient aortic stenosis and preserved left ventricular ejection fraction. Am J Cardiol 2014;114:1722-8.

46 Berti $\mathrm{D}$, Moors $\mathrm{E}$, Moons $\mathrm{P}$, et al. Prevalence and antithrombotic management of atrial fibrillation in hospitalised patients. Heart 2015;101:884-93.

47 Stamboul K, Zeller M, Fauchier L, et al. Prognosis of silent atrial fibrillation after acute myocardial infarction at 1-year follow-up. Heart 2015;101:864-9. 
48 Stamboul K, Zeller M, Fauchier L, et al. Incidence and prognostic significance of silent atrial fibrillation in acute myocardial infarction. Int J Cardiol 2014;174:611-17.

49 Sanna T, Diener HC, Passman RS, et al. Cryptogenic stroke and underlying atrial fibrillation. N Engl J Med 2014;370:2478-86.

50 Lankveld TA, Zeemering S, Crijns HJ, et al. The ECG as a tool to determine atrial fibrillation complexity. Heart 2014;100:1077-84.

51 Samol A, Masin M, Gellner R, et al. Prevalence of unknown atrial fibrillation in patients with risk factors. Europace 2013;15:657-62.

52 McManus DD, Lee J, Maitas 0, et al. A novel application for the detection of an irregular pulse using an iPhone 45 in patients with atrial fibrillation. Heart Rhythm 2013:10:315-19.

53 Lee J, Reyes BA, McManus DD, et al. Atrial fibrillation detection using an iPhone 4S. IEEE Trans Biomed Eng 2013;60:203-6.

54 Lau JK, Lowres N, Neubeck L, et al. iPhone ECG application for community screening to detect silent atrial fibrillation: a novel technology to prevent stroke. Int J Cardiol 2013;165:193-4.

55 Keach JW, Bradley SM, Turakhia MP, et al. Early detection of occult atrial fibrillation and stroke prevention. Heart 2015;101:1097-102.

56 Svennberg E, Engdahl J, Al-Khalili F, et al. Mass screening for untreated atrial fibrillation: the STROKESTOP study. Circulation 2015;131:2176-84.

57 Aronsson M, Svennberg E, Rosenqvist M, et al. Cost-effectiveness of mass screening for untreated atrial fibrillation using intermittent ECG recording. Europace 2015;17:1023-9.

58 Bax JJ, Marsan NA, Delgado V. Non-invasive imaging in atrial fibrillation: focus on prognosis and catheter ablation. Heart 2015;101:94-100.

59 Haeusler KG, Wilson D, Fiebach JB, et al. Brain MRI to personalise atrial fibrillation therapy: current evidence and perspectives. Heart 2014;100:1408-13.

60 Kobayashi A, Iguchi M, Shimizu S, et al. Silent cerebral infarcts and cerebral white matter lesions in patients with nonvalvular atrial fibrillation. J Stroke Cerebrovasc Dis 2012;21:310-17

61 Cha MJ, Park HE, Lee MH, et al. Prevalence of and risk factors for silent ischemic stroke in patients with atrial fibrillation as determined by brain magnetic resonance imaging. Am J Cardiol 2014;113:655-61.

62 Ovbiagele B, Saver JL, Sanossian N, et al. Predictors of cerebral microbleeds in acute ischemic stroke and TIA patients. Cerebrovasc Dis 2006;22:378-83.

63 Cuculich PS, Wang Y, Lindsay BD, et al. Noninvasive characterization of epicardial activation in humans with diverse atrial fibrillation patterns. Circulation 2010;122:1364-72.

64 Guillem MS, Climent AM, Millet J, et al. Noninvasive localization of maximal frequency sites of atrial fibrillation by body surface potential mapping. Cir Arrhythm Electrophysiol 2013;6:294-301.

65 Dogliotti A, Paolasso E, Giugliano RP. Current and new oral antithrombotics in non-valvular atrial fibrillation: a network meta-analysis of 79808 patients. Heart 2014; 100:396-405.

66 Caldeira D, Rodrigues FB, Barra M, et al. Non-vitamin K antagonist oral anticoagulants and major bleeding-related fatality in patients with atrial fibrillation and venous thromboembolism: a systematic review and meta-analysis. Heart 2015;101:1204-11.

67 Camm AJ, Lip GY, De Caterina R, et al. 2012 focused update of the ESC Guidelines for the management of atrial fibrillation: an update of the 2010 ESC Guidelines for the management of atrial fibrillation. Developed with the special contribution of the European Heart Rhythm Association. Eur Heart J 2012;33:2719-47.

68 January CT, Wann LS, Alpert JS, et al. 2014 AHA/ACC/HRS guideline for the management of patients with atrial fibrillation: a report of the American College of Cardiology/American Heart Association Task Force on practice guidelines and the Heart Rhythm Society. Circulation 2014;130:e199-267.

69 De Caterina R, Ammentorp B, Darius $\mathrm{H}$, et al. Frequent and possibly inappropriate use of combination therapy with an oral anticoagulant and antiplatelet agents in patients with atrial fibrillation in Europe. Heart 2014;100:1625-35.

70 Dinh T, Baur LH, Pisters R, et al. Aspirin versus vitamin $\mathrm{K}$ antagonist treatment guided by transoesophageal echocardiography in patients with atrial fibrillation: a pilot study. Heart 2014;100:563-8.
71 Vadmann $\mathrm{H}$, Nielsen PB, Hjortshoj SP, et al. Atrial flutter and thromboembolic risk: a systematic review. Heart 2015;101:1446-55.

72 Phan K, Xie A, La Meir M, et al. Surgical ablation for treatment of atrial fibrillation in cardiac surgery: a cumulative meta-analysis of randomised controlled trials. Heart 2014; 100:722-30.

73 Providencia R, Albenque JP, Combes S, et al. Safety and efficacy of dabigatran versus warfarin in patients undergoing catheter ablation of atrial fibrillation: a systematic review and meta-analysis. Heart 2014; 100:324-35.

74 Steinberg BA, Hasselblad V, Atwater BD, et al. Dabigatran for periprocedural anticoagulation following radiofrequency ablation for atrial fibrillation: a meta-analysis of observational studies. J Interv Card Electrophysio/ 2013;37:213-21.

75 Bin Abdulhak AA, Khan AR, Tleyjeh IM, et al. Safety and efficacy of interrupted dabigatran for peri-procedural anticoagulation in catheter ablation of atrial fibrillation: a systematic review and meta-analysis. Europace 2013;15:1412-20.

76 Kornej J, Hindricks G, Banerjee A, et al. Changes in renal function after catheter ablation of atrial fibrillation are associated with CHADS2 and CHA2DS2-VASc scores and arrhythmia recurrences. Heart 2015;101:126-31.

77 Bohm M, Ezekowitz MD, Connolly SJ, et al. Changes in renal function in patients with atrial fibrillation: an analysis from the RE-LY trial. J Am Coll Cardiol 2015;65:2481-93.

78 Takahashi Y, Takahashi A, Kuwahara T, et al. Renal function after catheter ablation of atrial fibrillation. Circulation 2011;124:2380-7.

79 Steinberg BA, Hammill BG, Daubert JP, et al. Periprocedural imaging and outcomes after catheter ablation of atrial fibrillation. Heart 2014;100:1871-7.

80 Kapa S, Martinez MW, Williamson EE, et al. ECG-gated dual-source CT for detection of left atrial appendage thrombus in patients undergoing catheter ablation for atrial fibrillation. J Interv Card Electrophysiol 2010;29:75-81.

81 Kettering K, Greil GF, Fenchel M, et al. Catheter ablation of atrial fibrillation using the Navx-/Ensite-system and a CT-/MRI-guided approach. Clin Res Cardiol 2009;98:285-96.

82 Ang R, Hunter RJ, Baker V, et al. Pulmonary vein measurements on pre-procedural CT/MR imaging can predict difficult pulmonary vein isolation and phrenic nerve injury during cryoballoon ablation for paroxysmal atrial fibrillation. Int I Cardiol 2015;195:253-8

83 Shigenaga Y, Kiuchi K, Okajima K, et al. Acquisition of the pulmonary venous and left atrial anatomy with non-contrast-enhanced MRI for catheter ablation of atrial fibrillation: Usefulness of two-dimensional balanced steady-state free precession. J Arrhythm 2015;31:189-95.

84 Shigenaga Y, Okajima K, Ikeuchi K, et al. Usefulness of non-contrast-enhanced MRI with two-dimensional balanced steady-state free precession for the acquisition of the pulmonary venous and left atrial anatomy pre catheter ablation of atrial fibrillation: comparison with contrast enhanced CT in clinical cases. J Magn Reson Imaging 2015. doi:10.1002/jmri.24990

85 McGann C, Akoum N, Patel A, et al. Atrial fibrillation ablation outcome is predicted by left atrial remodeling on MRI. Circ Arrhythm Electrophysiol 2014;7:23-30.

86 Hohnloser SH, Capucci A, Fain E, et al. ASymptomatic atrial fibrillation and Stroke Evaluation in pacemaker patients and the atrial fibrillation Reduction atrial pacing Trial (ASSERT). Am Heart J 2006;152:442-7.

87 Healey JS, Martin JL, Duncan A, et al. Pacemaker-detected atrial fibrillation in patients with pacemakers: prevalence, predictors, and current use of oral anticoagulation. Can J Cardiol 2013;29:224-8.

88 Lima C, Martinelli M, Peixoto GL, et al. Silent atrial fibrillation in elderly pacemaker users: a randomized trial using home monitoring. Ann Noninvasive Electrocardiol 2015.

89 Ricci RP, Pignalberi C, Landolina M, et al. Ventricular rate monitoring as a tool to predict and prevent atrial fibrillation-related inappropriate shocks in heart failure patients treated with cardiac resynchronisation therapy defibrillators. Heart 2014; 100:848-54.

90 Amara W. [ICD inappropriate shock for atrial fibrillation: use of home-monitoring for the prevention]. Ann Cardiol Angeiol (Paris) 2014;63:389-93. doi:10.1111/anec. 12294 